\title{
Caracteres Morfoestructurales de una Raza Caprina Recientemente Recuperada
}

\author{
Morphostructural Characters of a Recently Rescued Goat Breed
}

\author{
P.M. Parés-Casanova ${ }^{1,2}$, I. Kucherova ${ }^{1}$
}

\section{ReSUMEN}

Se estudiaron los caracteres cuantitativos, a partir del análisis de 19 variables morfoestructurales, de 23 hembras adultas de la población caprina «Cabra Catalana». Los animales muestreados procedían del mismo rebaño fundación, localizado en Catalunya, NE de España. Los animales presentaron un grado medio de homogeneidad y bajo de armonía. Se sugiere la necesidad de redactar el Prototipo Racial a fin de llegar a reconocer la raza como oficial.

Palabras clave: armonía, caprino, morfoestructura, raza local

\section{Abstract}

Quantitative characters based on 19 morphostructural variables from 23 adult females of the newly recovered «Cabra Catalana» goat were studied. The sample corresponded to animals located in the same foundational herd in Catalunya, NE Spain. Studied animals presented medium homogeneity and low harmony degrees. It is suggested that breed standards must be prepared in order to officially recognize this population.

Key words: harmony, caprine, local breed, morphostructure

\section{INTRODUCCIÓN}

La homologación de una raza supone que las características de ese animal quedan perfectamente registradas y existen unos estándares para los nuevos criadores. Sin reconocimiento oficial, una raza corre más riesgo de perderse al faltar los objetivos de tipificación y, por ende, de selección.

En el año 2006, apareció publicado «Catalans de Pèl i Ploma» (ISBN-10: 8496553-02-7), un libro de divulgación sobre

\footnotetext{
${ }^{1}$ Departament de Producció Animal, Universitat de Lleida, Catalunya, España

${ }^{2}$ E-mail: peremiquelp@prodan.udl.cat
} 
razas autóctonas de Cataluña. Uno de los capítulos se dedicó a la raça Catalana, que los autores presentaron como extinguida. Gran parte de la información fue extraída del «Diccionario de Agricultura, Zootecnia y Veterinaria», del insigne veterinario Rosell y Vilà, obra publicada en 1928, así como de fotografías originales de archivo, datadas de principios del siglo XX. Unos años después, Álvarez (2010) describe con detalle esta cabra, citando igualmente la obra de Rossell i Vilà. No obstante, Aparicio (1960) no cita esta población en su libro «Zootecnia especial: etnología compendiada».

En 2011, Santiago Álvarez localizó en Sant Salvador de Toló (cuenca de Tremp, Cataluña, España), ejemplares caprinos adultos que respondían marcadamente al tipo caprino descrito. A raíz de este encuentro, Gerard Batalla y Artur Bòria, integrantes del proyecto Cultures Trobades, de Slow Food, adquirieron 19 hembras y 2 machos. Este pequeño núcleo fundacional se halla actualmente localizado en Vilanova de Meià, en la serranía del Montsec, una zona en el centrooeste catalán de difícil orografía, suelo pobre y clima seco y cálido, donde cuenta con unos 50 ejemplares. Al tratarse de una población local y rústica, se le cría en régimen extensivo estricto, pastoreando en terrenos abruptos, de pastos pobres y clima extremo, estando perfectamente adaptada al medio.

Hace más de veinte años, la Organización de las Naciones Unidas para la Agricultura y la Alimentación (FAO) se pronunció sobre la importancia de conservar los recursos animales autóctonos, que se estaban perdiendo debido a la competencia de los animales industriales de importación, altamente productivos en condiciones óptimas, pero escasamente productivos en condiciones difíciles. Posteriormente, la FAO inició un vasto plan para recuperar y conservar especies y razas de animales (FAO, 2007). Hoy por hoy, es claro que las razas autóctonas están adaptadas y pueden ser productivas en su medio, donde, además, los productos de razas autóctonas se han revalorizado. Eso es algo que, por una parte, permite al consumidor acceder a productos de calidad $\mathrm{y}$, por otra, permite al ganadero competir con otras razas, a lo mejor más productoras en un principio, pero que al incrementar la producción, pierden la calidad.

El proceso para que una raza ganadera llegue a ser reconocida oficialmente como tal consta de tres pasos fundamentales. Este camino comienza con un primer reconocimiento de la agrupación racial. Luego, el reconocimiento de que, efectivamente, existe la raza en cuestión y, finalmente, la catalogación de la raza y su adscripción al grupo oportuno. La raza se identifica por una serie de caracteres étnicos determinados como «atributos, rasgos o signos de carácter permanente y heredables que permiten clasificar a los animales de una especie en razas». Más recientemente, el equipo taxonómico de referencia en Zoo-Etnología definió como raza a «un grupo homogéneo de animales domésti$\cos$ que poseen caracteres definidos e identificables (morfológicos, fanerópticos, morfoestructurales y fisiozootécnicos), transmisibles a la descendencia, que permiten distinguirlos fácilmente de otros grupos definidos de la misma manera dentro de la misma especie» (Herrera, 2002).

Oficialmente, el Ministerio de Agricultura, Pesca y Alimentación, España, considera como agrupación racial al grupo de animales con uniformidad de caracteres visibles y con transmisión de dichos caracteres a su descendencia, pero de homogeneidad no demostrada científicamente. Para que un grupo de animales alcance el estatus de agrupación racial, debe existir un colectivo adecuado, ya sea administración pública, entidad científica o agrupación de criadores, que solicite formalmente dicho reconocimiento ante el Comité de Razas del Ministerio. Esta solicitud debe incluir los datos referentes al origen e historia de la población de animales para los que se busca el reconocimiento, su censo y distribución geográfica y sus caracteres plásticos, fanerópticos, morfológicos, morfoestructurales y fisiozootécnicos. 
Este artículo pretende aportar datos biométricos que justifican la existencia de la Cabra Catalana -que hasta hace poco se creía extinguida- como población caprina diferenciada, y como base de su reconocimiento oficial.

\section{Materiales y Métodos}

Sobre un total de 23 hembras adultas catalanas, procedentes del único rebaño de recuperación, se tomaron 19 medidas zoométricas. Se determinó el peso corporal a través de una balanza digital; la alzada a la cruz, anchura de tórax y de grupa anterior con un bastón zoométrico; y el perímetro torácico, perímetro de la caña anterior, longitud auricular y longitud cornual, con una cinta métrica. Todas las medidas fueron tomadas por uno de los autores.

A partir de la imagen en plano lateral utilizando un tutor, se obtuvo por fotogrametría el resto de medidas lineales (longitud corporal y de la grupa, profundidad de tórax, longitud cefálica y facial, longitud de la espalda y del muslo) y diversos ángulos (espalda, brazo, grupa y muslo). La fotogrametría se realizó con el paquete UTHSCSA Image Tool v. 3.00.

Para la caracterización morfoestructural se ha utilizado la información suministrada por los estadísticos principales de las variables en estudio. La homogeneidad de la muestra fue valorada a través de los coeficientes de variación, lo que constituye per se un test de homogeneidad morfoestructural de los animales muestreados. Se realizó posteriormente una comparación con razas del tronco Celtibérico, a través de un análisis de clústeres, mediante el método de Ward y mediante un Análisis de Componentes Principales (ACP). La matriz con los coeficientes de correlación de Spearman entre todas las variables informó sobre el grado de armonía (Herrera, 2002). Se recurrió al coeficiente de Spearman ante la posibilidad de que alguna de las variables no presentase una distribución normal. Los cálculos estadísticos se realizaron con el programa PAST (Hammer et al., 2011).

\section{Resultados y Discusión}

En el Cuadro 1 se expresan los valores medios y los coeficientes de variación de las medidas zoométricas. Los coeficientes de variación más elevados se encuentran en las variables referidas a inclinación y longitud de extremidades, por lo que constituyen caracteres en los que se deben aplicar criterios de selección que aminoren la variabilidad encontrada. De todos modos, esta baja uniformidad podría haberse magnificado por la posición de los animales en el momento de las mediciones, puesto que un aplomo incorrecto provocaría error en su obtención (se trata de variables obtenidas por fotogrametría).

Del estudio con las medidas obtenidas de hembras adultas en rebaños de Blanca Celtibérica ubicados en Castilla La ManchaMurcia y Andalucía, y de Blanca de Rasquera (Carné, 2005), en valores a partir de nueve caracteres biométricos (alzada a la cruz, anchura de grupa anterior, longitudes cefálica, corporal, de grupa y facial, perímetro de caña anterior y torácico, y profundidad torácica) (Cuadro 2) se deduce que la Catalana ocupa un clúster separado (Fig. 1).

Del ACP para el estudio de las cuatro razas, se observa que las mayores descargas (85.5\% de la varianza total observada) corresponden a la alzada a la cruz, longitudes cefálica y facial, y profundidad torácica (Fig. 2). La Cabra Catalana se caracteriza, pues, por un tercio anterior más desarrollado que la Celtibérica y la Rasquera, y un tercio posterior menos desarrollado (menor longitud de la grupa); indicando que presenta un modelo morfoestructural distinto. Sin embargo, el análisis de correlación entre todas las variables indica que la Cabra Catalana presenta un grado medio de armonía morfoestructural, pues únicamente el $12.5 \%$ de los coeficien- 
Cuadro 1. Valores medios \pm desviación estándar, valores máximos y mínimos, y coeficientes de variación porcentual $(\mathrm{CV})$ de variables morfométricas de la Cabra Catalana

\begin{tabular}{lcccr}
\hline & $\bar{x} \pm$ de & Mínimo & Máximo & $\begin{array}{r}\text { CV } \\
(\%)\end{array}$ \\
\hline Inclinación del brazo $\left({ }^{\circ}\right)$ & $38.6 \pm 5.71$ & 30.2 & 51.9 & 14.8 \\
Inclinación del muslo $\left({ }^{\circ}\right)$ & $36.5 \pm 6.77$ & 23.2 & 45.5 & 18.5 \\
Inclinación de la espalda $\left(^{\circ}\right)$ & $59.1 \pm 6.75$ & 46.7 & 69.2 & 11.4 \\
Inclinación de la grupa $\left({ }^{\circ}\right)$ & $41.3 \pm 7.39$ & 28.6 & 54.2 & 17.9 \\
Alzada a la cruz $(\mathrm{cm})$ & $79.9 \pm 3.86$ & 72.7 & 86.2 & 4.8 \\
Anchura de grupa anterior $(\mathrm{cm})$ & $15.8 \pm 1.02$ & 14.0 & 18.0 & 6.4 \\
Anchura torácica $(\mathrm{cm})$ & $17.7 \pm 1.66$ & 15.0 & 21.0 & 9.4 \\
Longitud cefálica $(\mathrm{cm})$ & $28.5 \pm 1.93$ & 24.4 & 32.0 & 6.8 \\
Longitud cornual $(\mathrm{cm})$ & $41.0 \pm 5.32$ & 30.0 & 52.5 & 13.0 \\
Longitud corporal $(\mathrm{cm})$ & $76.5 \pm 5.47$ & 66.7 & 89.8 & 7.2 \\
Longitud de la espalda $(\mathrm{cm})$ & $26.0 \pm 2.76$ & 19.3 & 30.7 & 10.6 \\
Longitud de la grupa $(\mathrm{cm})$ & $19.6 \pm 1.96$ & 16.7 & 23.8 & 10.0 \\
Longitud del muslo $(\mathrm{cm})$ & $22.3 \pm 2.36$ & 18.5 & 28.4 & 10.6 \\
Longitud facial $(\mathrm{cm})$ & $16.4 \pm 1.35$ & 14.3 & 18.8 & 8.3 \\
Longitud auricular $(\mathrm{cm})$ & $16.5 \pm 1.48$ & 13.0 & 19.0 & 9.0 \\
Perímetro de la caña anterior $(\mathrm{cm})$ & $9.2 \pm 0.54$ & 8.0 & 10.5 & 5.9 \\
Perímetro torácico $(\mathrm{cm})$ & $88.2 \pm 6.87$ & 61.5 & 98.0 & 7.8 \\
Peso corporal $(\mathrm{kg})$ & $56.7 \pm 6.52$ & 42.3 & 70.2 & 11.5 \\
Profundidad torácica $(\mathrm{cm})$ & $40.2 \pm 2.58$ & 35.5 & 44.9 & 6.4 \\
\hline
\end{tabular}

Cuadro 2. Medidas lineales (alzada a la cruz, anchura de grupa posterior, longitud cefálica, corporal, de grupa, y facial, perímetro de caña anterior y torácico, y profundidad torácica) de Cabra Catalana, Blanca Celtibérica de Castilla La Mancha-Murcia y Andalucía, y Blanca de Rasquera, en hembras adultas (medidas en centímetros)

\begin{tabular}{|c|c|c|c|c|c|c|c|c|c|}
\hline $\begin{array}{l}\widetilde{\widetilde{N}} \\
\widetilde{\widetilde{U}}\end{array}$ & $\begin{array}{l}\stackrel{N}{J} \\
\dot{U} \\
\dot{U}\end{array}$ & 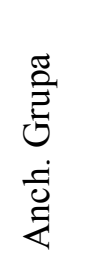 & 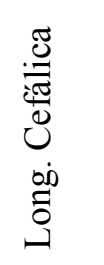 & 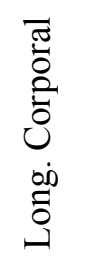 & 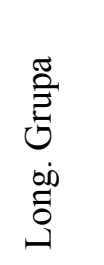 & 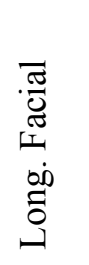 & 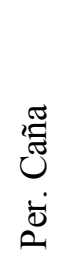 & 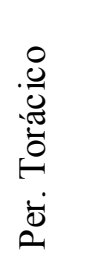 & 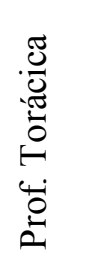 \\
\hline Catalana & 79.9 & 15.8 & 28.5 & 76.5 & 19.6 & 16.4 & 9.2 & 88.2 & 40.2 \\
\hline Castilla La Mancha-Murcia & 72.9 & 14.9 & 23.9 & 76.4 & 21.9 & 14.2 & 8.6 & 84.9 & 33.6 \\
\hline Andalucía & 69.1 & 11.9 & 22.7 & 73.9 & 21.2 & 12.8 & 9 & 86.3 & 31.1 \\
\hline Blanca de Rasquera & 71.9 & 15.8 & 23.3 & 71.1 & 20.5 & 13.8 & 9.4 & 87.4 & 33.3 \\
\hline
\end{tabular}


Cuadro 3. Coeficientes de correlación de Spearman para 18 variables zoométricas (longitud cornual excluida). Valores $\mathrm{p}$ en la diagonal superior, y valores absolutos $>0.6$ en la diagonal inferior

\begin{tabular}{|c|c|c|c|c|c|c|c|c|c|c|c|c|c|c|c|c|c|c|}
\hline & 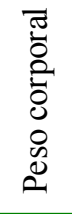 & 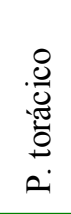 & 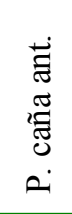 & 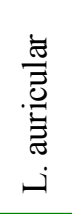 & 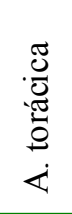 & 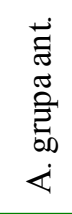 & 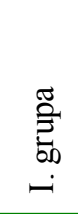 & $\begin{array}{l}\stackrel{N}{Z} \\
\text { ¿ } \\
\dot{Z}\end{array}$ & 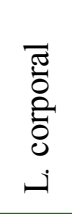 & 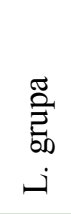 & 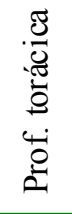 & 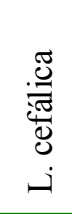 & 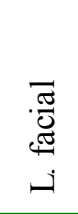 & $\begin{array}{l}\frac{\tilde{J}}{\tilde{\pi}} \\
\frac{\tilde{O}}{0} \\
\dot{0} \\
\dot{1}\end{array}$ & $\begin{array}{l}\frac{0}{\tilde{N}} \\
\stackrel{\Xi}{\Xi} \\
\dot{\lrcorner}\end{array}$ & 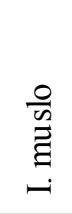 & 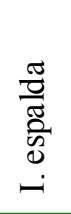 & 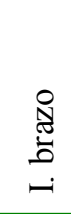 \\
\hline $\begin{array}{l}\text { Peso } \\
\text { corporal }\end{array}$ & - & 002 & 48 & .290 & .001 & .810 & .280 & .205 & .170 & .625 & .020 & .409 & .177 & .506 & .101 & .333 & .441 & .683 \\
\hline $\begin{array}{l}\text { Per. } \\
\text { torácico }\end{array}$ & .620 & - & .132 & .871 & .016 & .990 & .015 & .052 & .010 & .579 & .007 & .344 & .226 & .304 & .526 & .108 & .427 & .930 \\
\hline $\begin{array}{l}\text { Per. caña } \\
\text { ant. }\end{array}$ & & & - & .151 & .384 & .493 & .084 & .005 & .035 & .816 & .031 & .000 & .109 & .869 & .508 & .010 & .814 & .144 \\
\hline $\begin{array}{l}\text { Long. } \\
\text { Auricular }\end{array}$ & & & & - & .926 & .877 & .984 & .698 & .132 & .058 & .993 & .691 & .437 & .013 & .829 & .265 & .232 & .181 \\
\hline $\begin{array}{l}\text { A. } \\
\text { torácica }\end{array}$ & .648 & & & & - & .774 & .002 & .978 & .079 & .714 & .353 & .570 & .601 & .778 & .031 & .103 & .041 & .628 \\
\hline $\begin{array}{l}\text { A. grupa } \\
\text { anterior }\end{array}$ & & & & & & - & .942 & .669 & .866 & .255 & .836 & .508 & .583 & .327 & .726 & .428 & .886 & .825 \\
\hline I. grupa & & & & & & & - & .526 & .079 & .996 & .612 & .508 & .491 & .925 & .004 & .010 & .063 & .333 \\
\hline Al. cruz & & & & & & & & - & .018 & .268 & .005 & .001 & .002 & .185 & .568 & .460 & .599 & .568 \\
\hline $\begin{array}{l}\text { L. } \\
\text { corporal }\end{array}$ & & & & & & & & & - & .023 & .001 & .033 & .717 & .000 & .008 & .408 & .024 & .252 \\
\hline L. grupa & & & & & & & & & & - & .235 & .500 & .466 & .090 & .146 & .065 & .979 & .301 \\
\hline $\begin{array}{l}\text { Prof. } \\
\text { torácica }\end{array}$ & & & & & & & & .840 & .641 & & - & .003 & .004 & .016 & .129 & .654 & .730 & 1.00 \\
\hline $\begin{array}{l}\text { L. } \\
\text { cefálica }\end{array}$ & & & .680 & & & & & .625 & & & & - & .012 & .349 & .590 & .483 & .761 & .193 \\
\hline L. facial & & & & & & & & .601 & & & & & - & .670 & .771 & .430 & 1.00 & .412 \\
\hline $\begin{array}{l}\text { L. } \\
\text { espalda }\end{array}$ & & & & & & & & & .691 & & & & & - & .256 & 690 & .081 & .075 \\
\hline L. muslo & & .741 & & & & & & & & & & & & & - & .342 & .312 & .964 \\
\hline I. muslo & & & & & & & & & & & & & & & & - & .076 & .246 \\
\hline I. espalda & & & & & & & & & & & & & & & & & - & .043 \\
\hline I. brazo & & & & & & & & & & & & & & & & & & - \\
\hline
\end{tabular}

tes de correlación obtenidos entre las diferentes variables resultaron positivos y significativos (Cuadro 3). Ello puede deberse muy probablemente al variopinto origen genético (no de rebaño) de los animales estudiados, que pertenecen todos al núcleo fundacional estudiado.

\section{Conclusiones}

La caracterización morfológica y morfoestructural realizada en esta muestra de cabras catalanas indica que los animales ofrecen un grado medio de homogeneidad, 


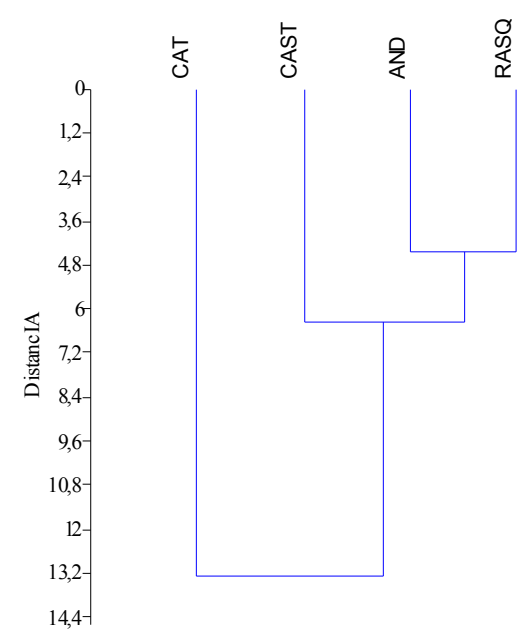

Figura 1. Dendrograma de Ward obtenido de la comparación de la Cabra Catalana (CAT) con la Blanca Celtibérica de Castilla La Mancha-Murcia (CAST), Blanca Celtibérica de Andalucía (AND) y Blanca de Rasquera (RASQ), a partir de nueve caracteres biométricos (alzada a la cruz, profundidad torácica, anchura de grupa posterior, longitudes corporal, de grupa, cefálica y facial, perímetro torácico y de caña anterior). Coeficiente cofenético $=0.922$ pero bajo de armonía, por lo que sería muy positiva la aplicación de un criterio de selección para la recría. Esto implica la redacción de un prototipo racial, que sería el segundo paso para el reconocimiento oficial de esta cabra como raza.

\section{Literatura Citada}

1. Álvarez S. 2010. La raza caprina Catalana. Feagas 36: 104-112.

2. Aparicio G. 1960. Zootecnia especial: etnología compendiada. Córdova: Ed. Imprenta Moderna. $465 \mathrm{p}$.

3. Carné S. 2005. La Cabra Blanca de Rasquera: caracterització estructural de les explotacions i estudi morfològic de la raça. Trabajo doctoral. Barcelona: Universitat Autònoma de Barcelona. $114 \mathrm{p}$.

4. [FAO] Organización de las Naciones Unidas para la Agricultura y la Alimentación. 2007. Plan de acción mundial sobre los recursos zoogenéticos y la Declaración de Interlaken. Roma: FAO. 52 p.

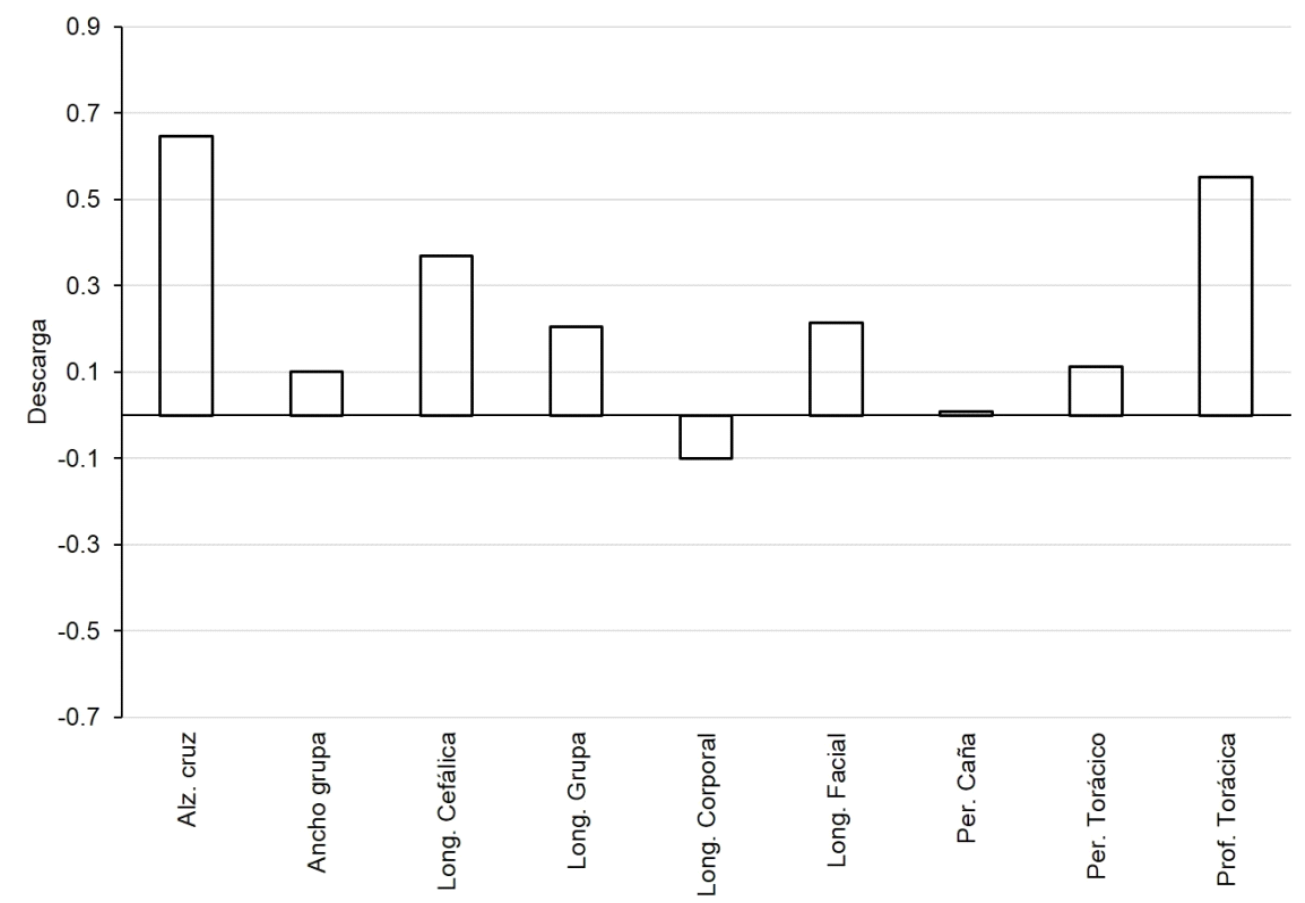

Figura 2. Descargas para el Componente Principal (85.5\% de la varianza total observada) de los datos biométricos correspondientes a Cabra Catalana con la Blanca Celtibérica de Castilla, La Mancha-Murcia, Blanca Celtibérica de Andalucía y Blanca de Rasquera. 
5. Hammer O, Harper DAT, Ryan PD. 2001. PAST: Paleontological statistics software package for education and data analysis. Palaeontologia Electronica 4(1). [Internet]. Disponible: http://palaeoelectronica.org/2001_1/past/ issue1_01.html

6. Herrera M. 2002. Criterios Etnozootécnicos para la definición de poblacio- nes animales. En: Actas V Congreso Ibérico sobre Recursos Genéticos Animales. Madrid: INIA. p 41-48.

7. Matons A, Rosell y Vila M. 1928. Diccionario de agricultura, zootecnia y veterinaria. Salvat Ed. Barcelona. 133 p.

8. Parés PM, Francesch A, Jordana J, Such $X$. 2006. Catalans de Pèl i Ploma. Barcelona: Lynx Edicions. 282 p. 